\title{
Clinical Profile of Bloodstream Infections in Covid-19 Patients: A Retrospective Cohort Study
}

\author{
Naveenraj Palanisamy \\ All India Institute of Medical Sciences Jodhpur \\ Nakka Vihari \\ All India Institute of Medical Sciences Jodhpur \\ Durga Shankar Meena ( $\nabla$ dsmims14@gmail.com) \\ All India Institute of Medical Sciences Jodhpur
}

\section{Deepak Kumar}

All India Institute of Medical Sciences Jodhpur

\section{Naresh Midha}

All India Institute of Medical Sciences Jodhpur

\section{Vibhor Tak}

All India Institute of Medical Sciences Jodhpur

\section{Ankur Sharma}

All India Institute of Medical Sciences Jodhpur

\section{Gopal Krishana Bohra}

All India Institute of Medical Sciences Jodhpur

\section{Nikhil Kothari}

All India Institute of Medical Sciences Jodhpur

\section{Naveen Dutt}

All India Institute of Medical Sciences Jodhpur

\section{Pradeep Kumar Bhatia}

All India Institute of Medical Sciences Jodhpur

Mahendra Kumar Garg

All India Institute of Medical Sciences Jodhpur

\section{Sanjeev Misra}

All India Institute of Medical Sciences Jodhpur

\section{Research Article}

Keywords: Covid-19, pneumonia, ICU, antimicrobial resistance, BSIs, Acinetobacter baumannii

Posted Date: June 22nd, 2021

DOI: https://doi.org/10.21203/rs.3.rs-614387/v1

License: (c) (i) This work is licensed under a Creative Commons Attribution 4.0 International License. Read Full License 


\section{Abstract}

Background: Bloodstream infections (BSIs) are emerging cause of significant morbidity and mortality in severe Corona virus disease (Covid-19). We aimed to assess the prevalence, clinical profile and outcome of BSIs in critically ill Covid-19 disease.

Material and Methods: This was a single-centre retrospective study conducted at a tertiary care hospital in Western India. All the patients (age >18 years) with reverse-transcription polymerase chain reaction (RT-PCR) confirmed Covid-19 pneumonia admitted in Covid intensive care unit (ICU) between September 2020 to February 2021 were included. Hospital electronic records were searched for demographic data, time of bloodstream infection since admission, clinical profile, antimicrobial resistance pattern and clinical outcome of all patients who developed BSIs.

Results: Out of 750 patients admitted in Covid ICU, 8.5\% developed secondary BSIs. All severe Covid-19 pneumonia patients developed BSIs succumbed to illness. The major proportion of BSIs were gram-negative pathogens $(53 / 64,82.8 \%)$. Acinetobacter baumannii was the commonest isolate followed by Klebsiella pneumoniae (32.8\% and $21.9 \%$ respectively). Multidrug-resistance microorganisms (MDRO) were found in $57.8 \%$ of the cases. The majority of MDRO belonged to K. pneumoniae and Enterococcus groups. The proportion of gram-negative bacteria resistant to carbapenems was $47.2 \%(25 / 53)$.

Conclusion: BSIs in severe Covid-19 patients carries a substantial mortality, which is a cause for concern. Timely initiation of empirical antibiotics and prompt de-escalation are vital to improve the outcome. At the same time, strict compliance of infection control practices should be accomplished to reduce the occurrence of MDRO.

\section{Background}

Covid-19 pandemic is an ongoing public health crisis causing death of more than three million person worldwide at the end of May 2021 [1]. Critical Covid illness is reported in around 5\% of the cases, which requires intensive care admission [2]. Case-fatality rate is highly variable (1.39 to $14 \%$ ) depending on the demography of infection [3]. During the course of hospitalization, it is difficult to predict the secondary bacterial infections, which warrant the use of empirical antimicrobials in patients with severe Covid-19 infection. There are recent reports showing conflicting results regarding the prevalence of secondary bacterial infections (ranges from 14.3 to $67.7 \%$ ), particularly bloodstream infections [4-8]. Furthermore, there is a scarcity of data from India regarding the BSIs and their impact on mortality in Covid-19. The knowledge of the precise burden of BSIs and their microorganism profile is essential so that empirical antimicrobial can be used judiciously in critically ill Covid-19 pneumonia patients. We aim to assess the prevalence, clinical profile, risk factors, frequency and distribution of microorganism, antimicrobial susceptibility and clinical outcome in severe Covid-19 with BSIs.

\section{Materials And Methods}

This retrospective observational study was carried out at a tertiary care centre in western Rajasthan, India which was also a dedicated Covid-ICU referral centre. From September 2020 to February 2021, all patients with confirmed Covid-19 positive (RT-PCR positive on nasopharyngeal or oropharyngeal swab) who were admitted in Covid-19 ICU were included. Those patients who already had BSIs before hospitalization in our center were excluded. The study was approved by the institutional ethical committee (reference no -AlIMS/IEC/2020/3174). BSIs were defined as the presence of at least one positive blood culture drawn that elicit or have elicited an inflammatory response characterized by the alteration of clinical, laboratory and hemodynamic parameters [9]. For skin contaminants (like coagulase negative Staphylococcus, at least 2 consecutive blood culture for the same pathogen were considered as BSIs. Isolation of similar micro-organism from blood stream within 14 days were not analyzed.

All electronic health records were searched and following clinical data were extracted: Demographic parameters (age, gender), risk factors for hospital-acquired BSIs like indwelling catheters, mechanical ventilation, use of immunosuppressants (corticosteroids or Tocilizumab for primary disease), co-morbidities (end stage renal disease, chronic liver disease, or malignancy), use of antibiotics, timing of blood culture drawn, duration of ICU stay, and outcome of patients. Hematological and biochemical parameters were collected which include hemoglobin, total leukocyte count, platelet count, c-reactive protein (CRP), procalcitonin, and lactate dehydrogenase (LDH). Quick sequential organ failure assessment (q-SOFA) score at baseline (at the time of ICU admission) was collected for each patient. 


\section{Identification of BSIs and antimicrobial susceptibility pattern}

Eight to ten $\mathrm{ml}$ of blood samples were collected aseptically and were inoculated into the BACTEC (BD, Biomerieux, France) bottles. The bottles were then loaded onto the automated system and incubated for five days minimum. Upon flagging positive, the bottles were taken out of the instrument and the time to detection (TTD) was noted. All those bottles which did not flag positive within five days were considered sterile. The positively flagged bottles were subjected to Gram's stain and culture onto blood agar and MacConkey agar. After 16 to 18 hours of incubation at $37^{\circ} \mathrm{C}$, the cultures were read for the growth of colonies and the Gram's stain and catalase tests were performed for further work-up. Antibiotic susceptibility testing was performed by Kirby Bauer's disk diffusion method. Zone of inhibition compared to the criteria set by the Clinical and Laboratory Standards Institute (CLSI) [10]. Multidrug resistant organism (MDRO) was defined as acquired non-susceptibility to at least one agent in three or more antimicrobial categories [10].

\section{Statistical Analysis}

Data analysis was performed using SPSS software, version 20.0 (IBM Corp, Armonk, NY). Descriptive data were summarized and tabulated with continuous variables in the form of mean \pm standard deviation, Median (inter quartile range) and categorical data in the form of percentages or frequencies. Linear regression model was used to highlight the association between day of culture positivity (duration from the day of hospitalization to blood culture positivity) and mortality. Coefficient of determination (R square) was calculated to show the percentage of variation in mortality attributed to day of culture positivity.

\section{Results}

During the study period of 6 months (September 2020 to February 2021), 750 patients with RT-PCR confirmed Covid-19 were admitted in ICU. Overall, 8.5\% patients (64/750) developed BSIs. Mortality rate was $100 \%$ (64) in BSIs group, and $33.8 \%$ (232) in nonBSIs Covid-19 group. Demographic and clinical characteristics of all patients with BSIs (64 patients) were summarized in Table-1. The median age of all patients was 65 years (IQR 54-70). The majority of patients were male (65.6\%). The median quick SOFA score (qSOFA) was 2.

Among the various co-morbidities, hypertension, diabetes, ischemic heart disease and ESRD (end stage renal disease) were common $(50 \%, 43.8 \%, 10.9 \%, 9.4 \%$ respectively). Corticosteroids were used in all patients, including non-BSIs patients. Seven patients received tocilizumab for Covid-19 pneumonia and developed BSIs. Two patients were renal transplant recipients and receiving tacrolimus during hospitalization. Among invasive devices, indwelling catheter and endotracheal tube $(71.9 \%$ and $68.8 \%)$ were most common. Biochemical parameters showed significant elevated CRP (C reactive protein) and procalcitonin levels (median CRP $128 \mathrm{mg} / \mathrm{L}$ and procalcitonin $5 \mathrm{ng} / \mathrm{ml}$, Table-1). The median time from ICU admission to the first BSI episode was 8 days (IQR, 4-11.8 days). The patients who died in hospital spent a median of 11.5 days (IQR, 7.25-16 days). Out of 64 patients of BSIs, 43 developed septic shock and 3 patients had features of septic encephalopathy. We analyzed the role of BSIs and sepsis in clinical outcome of Covid-19 patients admitted in ICU. Linear regression analysis showed positive correlation (Coefficient of determination, $R^{2}=74.2 \%, F i g .1$ ) between time to positivity of blood cultures and time to death (from day of hospitalization).

\section{Characteristics of microorganisms and antimicrobial resistance profile associated with BSIs}

All of the BSIs were monomicrobial in this study (table-1). A total of sixty-four isolates were identified from blood stream, the majority were gram-negative microorganism (53/64, 82.8\%). Among gram-positive microorganism, all isolates were from Enterococcus group $(11 / 64,17.2 \%)$. The most common gram-negative pathogens were Acinetobacter baumannii $(32.8 \%, 21 / 64)$, Klebsiella pneumoniae $(21.9 \%, 14 / 64)$, and E. coli $(7 / 64,10.9 \%)$. The remaining gram-negative microorganism (17.2\%) were Burkholderia cepaciae complex, Pseudomonas aeruginosa, and Elizabethkingia spp. (Fig. 2). None of the patients were associated with mixed BSIs in this analysis. Out of sixty-four patients, $57.8 \%$ patients were infected with multidrug-resistance microorganisms (MDRO). The majority of MDRO belonged to K. pneumoniae and Enterococcus spp. The proportion of gram-negative bacteria (GNB) resistant to carbapenems was $47.2 \%$ (25/53). Among isolated pathogens, highest resistance for Acinetobacter baumannii was observed with ceftriaxone (76.2\%), piperacillin-tazobactam (76.2\%) and fluoroquinolone (66.7\%). Colistin resistance was seen in only $9.5 \%$ of the $A$. baumanni isolates (Table-2). Klebsiella pneumoniae isolates showed resistance to majority of antibiotics. The highest resistance rate was showed against aztreonam (85.7\%), fluoroquinolone (78.6\%), piperacillin-tazobactam (78.6\%) and ceftriaxone 
(71.4\%). The majority of antibiotics were resistant to E. coli. All E. coli isolates were resistant to ceftriaxone, furthermore $85.7 \%$ isolates showed resistance to fluoroquinolone and cefepime. Enterococcus spp. showed maximum resistance for erythromycin, ampicillin and fluoroquinolone (90.9\%, 81.8\% and $81.8 \%$ respectively). In contrast, all isolates were susceptible to linezolid (Table-3).

\section{Discussion}

Severe Covid-19 pneumonia required prolonged hospital (ICU) stay, which predisposes them for secondary bacterial infections. Thus, the clinical outcome of severe Covid-19 illness also depends on propensity of secondary infections along with primary disease. Most evidences from literature which strengthened the aforementioned theory were originated during the 1918-19 Influenza pandemic. Secondary bacterial infections were the commonest cause of death during that pandemic $[9,11]$. Similar observations were derived from microbiological studies during the recent H1N1 Influenza pandemic [12]. The destruction of respiratory epithelial membrane, upregulation of various receptors through which bacteria can adhere to respiratory epithelium, impair function of neutrophils and macrophages secondary to release of cytokines like interferon gamma, IL-6, IL-10 and IL-17 were the different mechanism described for bacterial co-infections in viral pneumonia $[13,14]$. In addition, there is a SARS-Cov-2 mediated downregulation of immune function related gene expression $[15,16]$. Another theory is platelets driven intense immunosuppression which activates the biofilm resulting in secondary bacterial infections. The release of TGF- $\beta$ (transforming growth factor) and other proinflammatory cytokines from platelets is thought to be the trigger for immunosuppression $[17,18]$.

The emerging data regarding the secondary bacterial infections in Covid-19 illness are scarce and conflicting, even more so with BSIs. In this study, we found the overall prevalence of BSIs in ICU patients to be $8.5 \%$. A recent study from India found the prevalence of secondary infections to be 3.6\% [19]. However, in their report both hospital-acquired and community acquired cases were taken. In addition, apart from BSIs respiratory specimen were also analyzed which could be contaminants or colonizers. Another report by Khurana et al showed $13 \%$ prevalence of secondary infections [20]. Contrary to previous studies, Lai et al described the prevalence of BSIs up to $50 \%$ among non-survivors severe Covid-19 pneumonia patients [21]. Further large prospective studies are needed to determine the precise prevalence of BSIs in severe Covid-19 disease and its implications on outcome of patients. Gram-negative microorganism were predominant BSIs in this report. Similar observations were described by a multicentric study from India with a predominance of gram-negative pathogens (78\%) [19]. Conversely, Elabaddi et al and a few other studies reported the increase prevalence of gram-positive microorganism particularly Staphylococcus aureus (prevalence varies from 44-79.6\%) in Covid-19 ICU patients $[5,22]$. This heterogenicity in prevalence and distribution of microorganisms may attribute to different patient settings, number of patients on mechanical ventilation, duration of hospital stays and follow-up and isolation of pathogen from other specimens (like respiratory, urine and pus sample) in addition to BSIs. One interesting aspect of this report was the relatively high proportion of Enterococcus isolates as BSI and the majority were multidrug-resistant (81.8\% MDRO). Bonazetti et al proposed a theory that SARS CoV2 mediated disruption of gut barrier and bacterial translocation could be the trigger of increased BSIs, especially Enterococci spp. [4]. Genotypic analysis was not available at our Centre which would have provided some insight into this occurrence.

In this report, we found significantly elevated C-reactive protein and procalcitonin levels in patients who developed BSIs. These inflammatory markers could be vital for prediction of BSIs, empirical initiation of antibiotics in these patients will be prudent for better survival. Conversely, there are a few studies which reported the poor correlation of serum procalcitonin and CRP levels with bacterial co-infections $[5,22]$. Kreitmann et al reported that median CRP and procalcitonin did not differ significantly in patients with or without bacterial coinfections. (median procalcitonin, $0.4 \mathrm{vs} 0.72 \mathrm{ng} / \mathrm{ml}, \mathrm{CRP}, 182 \mathrm{vs} 159 \mathrm{mg} / \mathrm{L}$ ) [23]. Though we did not compare inflammatory markers with non-bacterial co-infection group, still the median procalcitonin level was $5 \mathrm{ng} / \mathrm{ml}$ which was significant in the context of BSIs. One of the highlights of this study was $100 \%$ mortality rate in severe Covid-19 pneumonia patients with BSIs. Previous studies have reported the mortality of $21-68 \%$ in this group of patients $[4,5,19,22]$. Disparity in mortality could be due to various factors; in our study all patients had a severe Covid-19 illness admitted in ICU, while previous studies also included patients admitted in non-ICU setting with mild to moderate illness. In earlier studies, respiratory, urine and local tissue samples were analyzed predominantly, which increases the probability of contaminants or colonizers. Furthermore, we followed all patients in their ICU stay till discharge or death for a precise outcome. Conversely, in some of the prospective studies follow-up time was brief or incomplete which might have exaggerated the survival rate. Comorbid conditions also played an important role in mortality with nearly half of the patients in this study having diabetes and hypertension. Few studies also showed high Covid-19 associated mortality in male gender $[24,25]$, which is important due to the high proportion of male patients $(65 \%)$ in our study. In this report, we found sepsis as a 
determining factor in clinical outcome. Soon after the onset of sepsis and isolation of the bloodstream pathogen, patients succumbed to illness.

Another interesting observation of this study was the high prevalence of $A$. baumannii (32.8\%). However, previous reports described the occurrence of $A$. baumanni in $<1 \%$ of the cases $[4,26]$. The other worrying aspect was the high proportion of carbapenem resistance in these isolates (nearly 50\%). This increase in prevalence and resistance could be explained by prolonged ICU stay, mechanical ventilation, use of central venous catheter and inadvertent use of carbapenem with antimicrobial selection pressure, which are the usual triggers [27-29]. In addition, incomplete compliance with infection control procedures (like hand hygiene, disinfection of hospital equipment and environment) is the other vital factor contributing to multidrug resistance organism infections. This study has a number of limitations; first this was a retrospective analysis which probably led to selection bias and inclusion of severe patients which may have impacted the clinical outcome. Secondly, data were from a single centre, which may preclude its generalized applicability. All patients received corticosteroids and other immunosuppressants resulting in high prevalence of secondary bacterial infections. We did not compare our findings with non-BSIs Covid-19 patients which could have represented the better clinical scenario. Lastly, lack of prospective design precluded the genotypic analysis of MDRO (A. baumannii), which is important in view of patient cross-contamination in ICU settings.

\section{Conclusions}

BSIs in severe Covid-19 are associated with worse outcome. We report a high prevalence of Acinetobacter baumannii BSIs in the ICU setting. We encourage the implementation of Antimicrobial stewardship and infection control practices which could be vital to reduce the secondary bacterial infections in Covid-19 illness. Further prospective studies are warranted to determine the precise burden of secondary infections and their impact on mortality and morbidity in Covid-19.

\section{Abbreviations}

BSIs: Bloodstream infections

Covid-19: Corona virus disease

RT-PCR: reverse-transcription polymerase chain reaction

ICU: intensive care unit

MDRO: Multidrug-resistance microorganisms

CRP: c-reactive protein

LDH: lactate dehydrogenase

q-SOFA: Quick sequential organ failure assessment

ESRD: end stage renal disease

GNB: gram-negative bacteria

TGF- $\beta$ : transforming growth factor- $\beta$

\section{Declarations}

\section{Ethics approval and consent to participate}

This study was approved by the institutional ethical committee of All India Institutes of Medical Sciences Jodhpur, Rajasthan (reference no - AllMS/IEC/2020/3174). The study was conducted in accordance with ethical principles for medical research involving human subjects as outline in the Declaration of Helsinki. Written informed consent was obtained from all participants and parents/legally authorized representative of minor participants involved in the study. 


\section{Funding}

This research did not receive any specific grant from funding agencies in the public, commercial, or not-for-profit sectors.

\section{Availability of Data and Materials}

The datasets used and/or analysed during the current study available from the corresponding author on reasonable request.

\section{Consent for publication}

Not applicable.

\section{Competing interests}

The authors declare that there are no competing interests.

\section{Authors' Contributions}

DK, DSM, NP, and VN developed the study conception and design. VT, NM, GKB, AS undertook data curation. DK, DSM, ND performed data analysis and interpretation. DSM, NK, NP, and VN drafted manuscript. PB, MKG, SM, ND conducted critical revision of the manuscript for important intellectual content. SM, MKG, and ND provided administrative, technical or material support. DSM is the guarantor of the study. The corresponding author attests that all listed authors meet the ICMJE criteria for authorship and that no other meeting the criteria have been omitted.

\section{Acknowledgements}

We thank all healthcare workers who took part in patients management.

\section{References}

1. WHO COVID-19 Dashboard. Geneva: World Health Organization, 2020. Available online: https://covid19.who.int/

2. Wu Z, McGoogan JM. Characteristics of and Important Lessons From the Coronavirus Disease 2019 (COVID-19) Outbreak in China: Summary of a Report of 72314 Cases From the Chinese Center for Disease Control and Prevention. JAMA. 2020;323:1239-1242.

3. Jain VK, lyengar K, Vaish A, Vaishya R. Differential mortality in COVID-19 patients from India and western countries. Diabetes Metab Syndr. 2020;14:1037-1041.

4. Bonazzetti C, Morena V, Giacomelli A, Oreni L, Casalini G, Galimberti LR, et al. Unexpectedly High Frequency of Enterococcal BSIs in Coronavirus Disease 2019 Patients Admitted to an Italian ICU: An Observational Study. Crit Care Med. 2021;49:e31-e40.

5. Giacobbe DR, Battaglini D, Ball L, Brunetti I, Bruzzone B, Codda G, et al. BSIs in critically ill patients with COVID-19. Eur J Clin Invest. 2020;50:e13319.

6. Langford BJ, So M, Raybardhan S, Leung V, Westwood D, MacFadden DR, et al. Bacterial co-infection and secondary infection in patients with COVID-19: a living rapid review and meta-analysis. Clin Microbiol Infect. 2020;26:1622-1629.

7. Vaillancourt M, Jorth P. The Unrecognized Threat of Secondary Bacterial Infections with COVID-19. mBio. 2020;11:e01806-20.

8. Ripa M, Galli L, Poli A, Oltolini C, Spagnuolo V, Mastrangelo A, et al; COVID-BioB study group. Secondary infections in patients hospitalized with COVID-19: incidence and predictive factors. Clin Microbiol Infect. 2021;27:451-457.

9. Brundage JF, Shanks GD. Deaths from bacterial pneumonia during 1918-19 influenza pandemic. Emerg Infect Dis. 2008;14:1193-9.

10. Magiorakos AP, Srinivasan A, Carey RB, Carmeli Y, Falagas ME, Giske CG, et al. Multidrug-resistant, extensively drug-resistant and pandrug-resistant bacteria: an international expert proposal for interim standard definitions for acquired resistance. Clin Microbiol Infect. 2012;18:268-81.

11. Morris DE, Cleary DW, Clarke SC. Secondary Bacterial Infections Associated with Influenza Pandemics. Front Microbiol. 2017;8:1041. 
12. Maclntyre CR, Chughtai AA, Barnes M, Ridda I, Seale H, Toms R, et al. The role of pneumonia and secondary bacterial infection in fatal and serious outcomes of pandemic influenza a(H1N1)pdm09. BMC Infect Dis. 2018;18:637.

13. Peltola VT, McCullers JA. Respiratory viruses predisposing to bacterial infections: role of neuraminidase. Pediatr Infect Dis J. 2004;23:S87-97.

14. Sun K, Metzger DW. Inhibition of pulmonary antibacterial defense by interferon-gamma during recovery from influenza infection. Nat Med. 2008;14:558-64.

15. Manna S, Baindara P, Mandal SM. Molecular pathogenesis of secondary bacterial infection associated to viral infections including SARS-CoV-2. J Infect Public Health. 2020;13:1397-1404.

16. Feldman C, Anderson R. The role of co-infections and secondary infections in patients with COVID-19. Pneumonia (Nathan). 2021;13:5.

17. Zhang F, Wang H, Wang X, Jiang G, Liu H, Zhang G, et al. TGF- $\beta$ induces M2-like macrophage polarization via SNAIL-mediated suppression of a pro-inflammatory phenotype. Oncotarget. 2016;7:52294-52306.

18. Fava RA, Casey TT, Wilcox J, Pelton RW, Moses HL, Nanney LB. Synthesis of transforming growth factor-beta 1 by megakaryocytes and its localization to megakaryocyte and platelet alpha-granules. Blood. 1990;76:1946-55.

19. Vijay S, Bansal N, Rao BK, Veeraraghavan B, Rodrigues C, Wattal C. Secondary Infections in Hospitalized COVID-19 Patients: Indian Experience. Infect Drug Resist. 2021;14:1893-1903

20. Khurana S, Singh P, Sharad N, Kiro VV, Rastogi N, Lathwal A. Profile of co-infections \& secondary infections in COVID-19 patients at a dedicated COVID-19 facility of a tertiary care Indian hospital: Implication on antimicrobial resistance. Indian J Med Microbiol. 2021;39:147-153.

21. Lai CC, Wang CY, Hsueh PR. Co-infections among patients with COVID-19: the need for combination therapy with non-anti-SARScoV-2 agents? J Microbiol Immunol Infect. 2020;53:505-12.

22. Elabbadi A, Turpin M, Gerotziafas GT, Teulier M, Voiriot G, Fartoukh M. Bacterial coinfection in critically ill COVID-19 patients with severe pneumonia. Infection. 2021;3:1-4

23. Kreitmann L, Monard C, Dauwalder O, Simon M, Argaud L. Early bacterial co-infection in ARDS related to COVID-19. Intensive Care Med. 2020;46:1787-9.

24. Jin JM, Bai P, He W, Wu F, Liu XF, Han DM. Gender Differences in Patients With COVID-19: Focus on Severity and Mortality. Front Public Health. 2020 Apr 29;8:152.

25. Yanez ND, Weiss NS, Romand JA, Treggiari MM. COVID-19 mortality risk for older men and women. BMC Public Health. 2020;20:1742.

26. Baiou A, Elbuzidi AA, Bakdach D, Zaqout A, Alarbi KM, Bintaher AA. Clinical characteristics and risk factors for the isolation of multi-drug-resistant Gram-negative bacteria from critically ill patients with COVID-19. J Hosp Infect. 2021;110:165-171.

27. Maragakis LL, Perl TM. Acinetobacter baumannii: epidemiology, antimicrobial resistance, and treatment options. Clin Infect Dis. 2008;46:1254-63.

28. Montrucchio G, Corcione S, Sales G, Curtoni A, De Rosa FG, Brazzi L. Carbapenem-resistant Klebsiella pneumoniae in ICUadmitted COVID-19 patients: Keep an eye on the ball. J Glob Antimicrob Resist. 2020;23:398-400.

29. Pascale R, Bussini L, Gaibani P, Bovo F, Fornaro G, Lombardo D. Carbapenem resistant bacteria in Intensive Care Unit during COVID-19 pandemic: multicenter before-after cross sectional study. Infect Control Hosp Epidemiol. 2021:1-25.

\section{Tables}

Table-1: Demographic, Clinical and Laboratory data of COVID-19 ICU patients with Blood stream infections 


\begin{tabular}{|c|c|}
\hline Parameters & Total Patients ( $\mathrm{n}=64)$ \\
\hline $\begin{array}{l}\text { Age (Median) } \\
\quad(\text { Mean } \pm \text { SD) }\end{array}$ & $\begin{array}{l}65 \text { years }(\mathrm{IQR}=54-70) \\
61.32 \pm 13.74 \text { years }\end{array}$ \\
\hline Male (\%) & $42(65.6)$ \\
\hline q SOFA Score (Median) & 2 \\
\hline \multicolumn{2}{|l|}{ Co-morbidities and Risk factors (\%) } \\
\hline $\begin{array}{l}\text { Hypertension } \\
\text { Diabetes Mellitus } \\
\text { Ischemic Heart Diseases } \\
\text { Immunosuppressants use } \\
\text { CKD/ESRD } \\
\text { Hypothyroidism } \\
\text { Stroke } \\
\text { COPD } \\
\text { Rheumatoid Arthritis } \\
\text { Chronic Liver Disease }\end{array}$ & $\begin{array}{l}32(50) \\
28(43.8) \\
7(10.9) \\
9(10.9) \\
6(9.4) \\
5(7.8) \\
4(6.4) \\
2(3.2) \\
2(3.2) \\
1(1.6)\end{array}$ \\
\hline \multicolumn{2}{|l|}{ Invasive Devices (insitu), (\%) } \\
\hline $\begin{array}{l}\text { Foleys Catheter } \\
\text { Endotracheal intubation }\end{array}$ & $\begin{array}{l}46(71.9) \\
44(68.8)\end{array}$ \\
\hline $\begin{array}{l}\text { Arterial line } \\
\text { Central venous line }\end{array}$ & $\begin{array}{l}21(32.8) \\
17(26.6)\end{array}$ \\
\hline $\begin{array}{l}\text { Laboratory parameters, Median ( } \\
\text { Hb (g/dl) } \\
\text { Total leukocyte counts }\left(10^{3} / \mathrm{ul}\right) \\
\text { Platelet count }\left(10^{3} / \mathrm{ll}\right) \\
\text { CRP }(\mathrm{mg} / \mathrm{L}) \\
\text { Procalcitonin (ng/ml) } \\
\text { AST (IU/L) } \\
\text { ALT (IU/L) } \\
\text { Blood Urea Nitrogen (mg/dl) } \\
\text { Creatinine }(\mathrm{mg} / \mathrm{dl})\end{array}$ & $\begin{array}{l}10(8.25-12) \\
16.5(11.2-22.8) \\
198(111-341) \\
128(97-171) \\
5(1.75-15.5) \\
44(29-134) \\
42(26-92) \\
100(56-130) \\
2(1-3)\end{array}$ \\
\hline \multicolumn{2}{|c|}{ Bacterial microorganism identified in Blood-stream } \\
\hline $\begin{array}{l}\text { Acinetobacter baumannii } \\
\text { Klebsiella Pneumoniae } \\
\text { Enterococcus Spp. } \\
\text { Eshcherechia Coli } \\
\text { Burkholderia Cepaciae Complex } \\
\text { Pseudomonas aeruginosa } \\
\text { Elizabethkingia Spp. }\end{array}$ & $\begin{array}{l}21(32.8) \\
14(21.9) \\
11(17.2) \\
7(10.9) \\
4(6.3) \\
4(6.3) \\
3(4.7)\end{array}$ \\
\hline
\end{tabular}

Table-2: Antibiotic resistance pattern of predominant Gram-negative blood stream isolates in Covid-19 ICU Patients (\%) 


\begin{tabular}{|lllllll|}
\hline Antibiotic & $\begin{array}{l}\text { Acinetobacter } \\
\text { baumannii } \\
(\mathbf{n = 2 1 )}\end{array}$ & $\begin{array}{l}\text { Klebsiella } \\
\text { pneumoniae } \\
(\mathbf{n = 1 4 )}\end{array}$ & $\begin{array}{l}\text { Psuedomonas } \\
\text { aeruginosa } \\
(\mathbf{n = 4})\end{array}$ & $\begin{array}{l}\text { Escherichia } \\
\text { coli } \\
(\mathbf{n}=7)\end{array}$ & $\begin{array}{l}\text { Burkholderia } \\
\text { cepacia } \\
\text { complex } \\
(\mathbf{n}=4)\end{array}$ & $\begin{array}{l}\text { Elizabethkingia } \\
\text { spp. } \\
(\mathbf{n}=3)\end{array}$ \\
\hline Ceftriaxone & 76.2 & 71.4 & NA & 100 & NA & NA \\
\hline Cefepime & 52.4 & 78.6 & 0 & 85.7 & NA & 66.6 \\
\hline Ceftazidime & NA & NA & NA & NA & 50 & NA \\
\hline Piperacillin-Tazobactam & 76.2 & 78.6 & 0 & 71.4 & NA & 33.3 \\
\hline Meropenem & 33.3 & 21.4 & 0 & 14.3 & 75 & 100 \\
\hline Colistin & 9.5 & 7.1 & 0 & 14.3 & 50 & 33.3 \\
\hline Ciprofloxacin/Levofloxacin & 66.7 & 78.6 & NA & 85.7 & 50 & NA \\
\hline Cefoperazone/sulbactam & NA & 71.4 & 0 & 57.1 & NA & NA \\
\hline Amikacin & 52.4 & 57.1 & 0 & 57.1 & NA & 66.6 \\
\hline Tigecycline & 0 & 0 & NA & 0 & NA & NA \\
\hline Aztreonam & NA & 85.7 & 50 & 71.4 & NA & 66.6 \\
\hline Imipenem & 9.5 & NA & 0 & 28.6 & NA & NA \\
\hline Ertapenem & 23.8 & 71.4 & NA & NA & NA & NA \\
\hline
\end{tabular}

Table - 3: Antibiotic resistance pattern of predominant Gram-positive blood stream isolates in Covid-19 ICU patients (\%)

\begin{tabular}{|ll|}
\hline Antibiotic & Enterococcus spp. $(\mathbf{N}=\mathbf{1 1})$ \\
\hline Ampicillin & 81.8 \\
\hline Tigecycline & 0 \\
\hline Ciprofloxacin & 81.8 \\
\hline Tetracycline & 54.5 \\
\hline Erythromycin & 90.9 \\
\hline Linezolid & 0 \\
\hline Teicoplanin & 18.1 \\
\hline Vancomycin & 18.1 \\
\hline
\end{tabular}

\section{Figures}




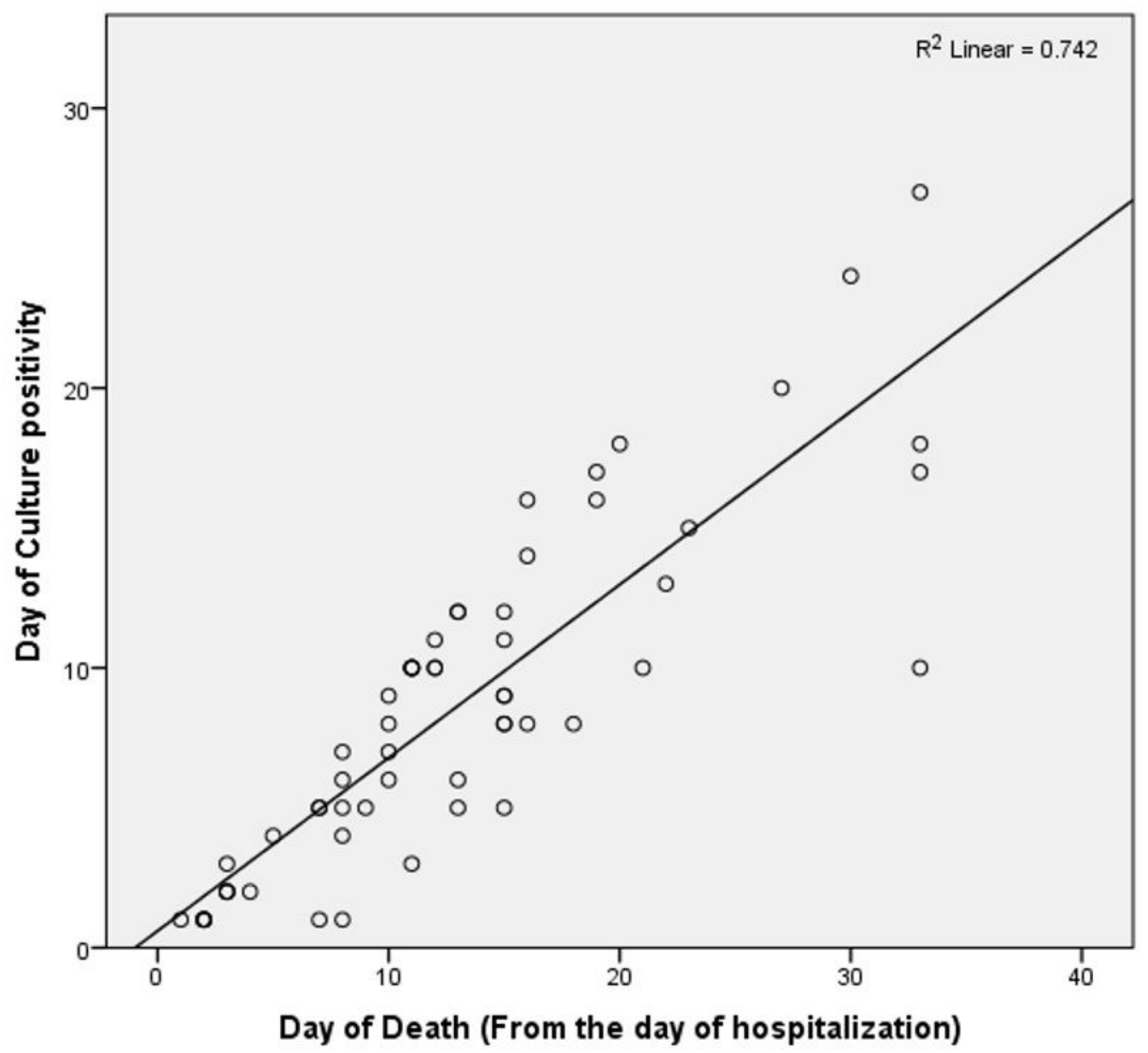

Figure 1

Linear regression graph showing positive correlation between day of culture positivity and day of mortality 


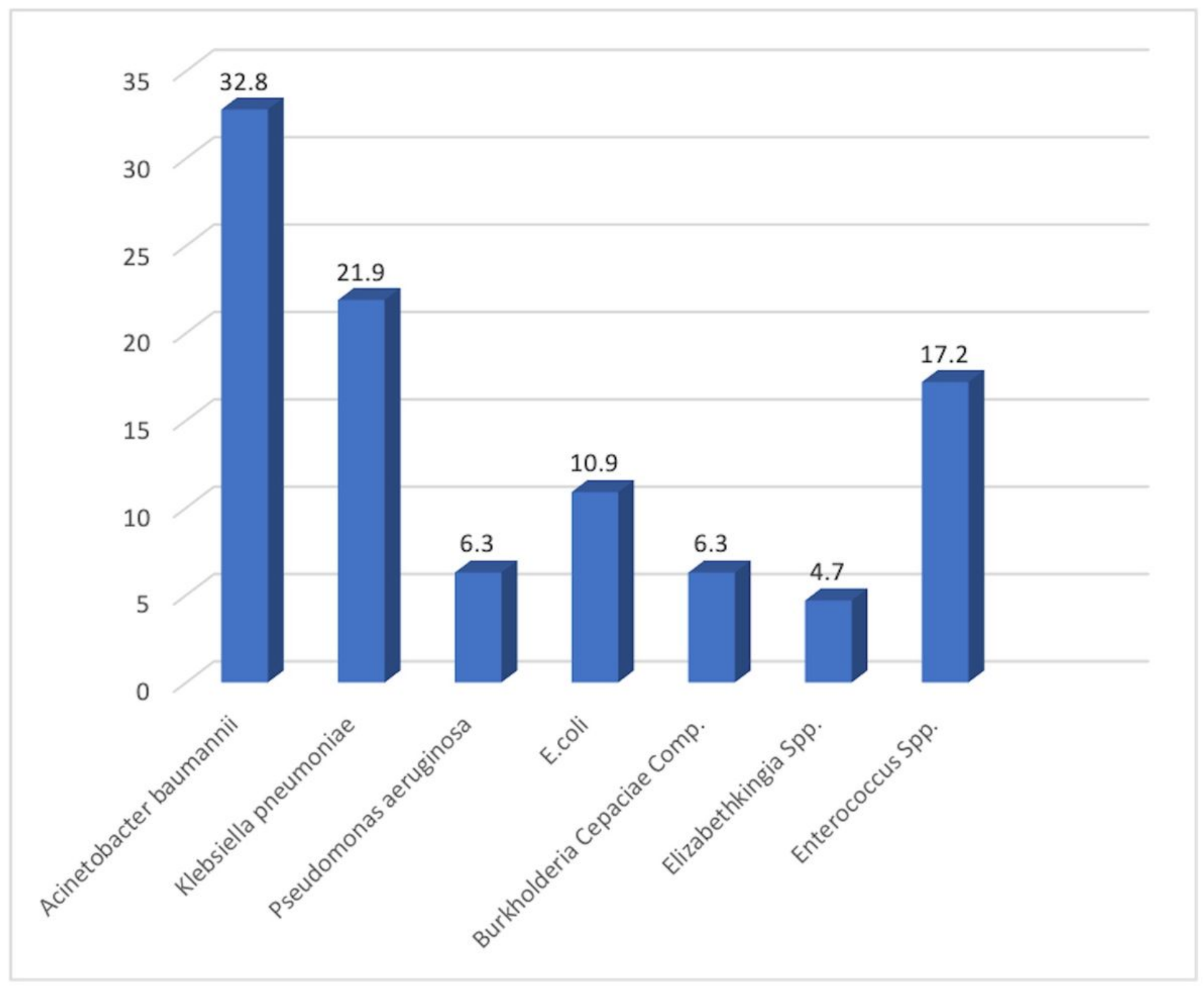

Figure 2

Frequency and distribution of pathogenic organism isolated from COVID-19 ICU patients (\%) 